\title{
THE NEXT GENERATION: AN 8-16 M SPACE TELESCOPE
}

\author{
Garth D. Illingworth \\ Lick Observatory/Board of \\ Studies in Astronomy and Astrophysics
}

\section{Introduction}

The Hubble Space Telescope is to be a long-lived observatory with wide-ranging spectroscopic and imaging capability in the UV and the visible, and in the near-IR as the second-generation instruments are implemented. HST will have a dramatic impact on our view of the universe. However, it is by no means premature to address the issue of its successor, even though HST has not yet been launched. We must look ahead with the realistic view that HST will degrade and will need to be replaced. The question that we must then address is:

What is the UV-Visible-IR Observatory that will follow HST?

I will make the case for this being an 8-16 m class telescope.

In developing the case for such a successor to HST, one that we might think of as being the Son of HST (or in this day, the Daughter of HST), we need to first focus on certain broad questions. We need to be clear in our own minds as to what the role of such a successor will be, and to elucidate clearly its scientific potential so that we can make the strongest possible case to:

- our colleagues and to the science community at large;

- to NASA, and to ESA and other space agencies, since I think that it is desirable that such a facility be international;

- to those who must budget and appropriate the funds in Congress, in the Administration, and in other governments.

The questions that we have to address are fivefold and are:

- Why do we need a successor to HST?

- What are the outstanding scientific objectives?

- What are the capabilities that we need?

- What is the successor to HST?

- Why do we need to start now?

While the answers to these questions may appear obvious to us, they are not to all our colleagues, nor to scientists in other fields, and certainly not to those being asked to fund such a major enterprise.

\section{The Case for a Successor}

Why do we need a successor to HST? To my mind there are two major reasons:

a) Continuity. HST is an observatory of wide-ranging capability. The scientific case for such capability will remain as strong in the future as it was in the seventies, and is now.

b) Discoveries. New technology will allow us to make a major step in the power of the next generation telescope, allowing us to open up new "discovery space" by a significant amount.

The argument (a) for continuing capability to carry out major programs and to support other missions is a strong one. It is my view that the development of scientific understanding is a mixture of both discovery and goal-oriented programs, and that these two aspects of scientific research play comparable and complementary roles. There is no doubt that we can make a strong case for continuity of access to the wide-ranging observational capabilities exemplified 
by HST. I am sure that any astronomer, particularly the observers, can imagine the impact on their research programs if access to their primary facility was cut off for 10-15 years. It would be devastating, yet this is what has happened in X-ray astronomy. The Einstein satellite had a dramatic impact in many areas. It offered us a tantalizing glimpse of data on many important problems - and then the door was shut, in most cases until AXAF (the Advanced $X$-ray Astrophysics Facility) flies in the mid-1990's. Clearly this is not the way to proceed. We need to work towards missions that, while not necessarily overlapping or contiguous, at least have hiatuses that are of the order of 5 years or less.

It is greatly to NASA's credit that they have recognized this. The development of the Great Observatories program is an excellent response to this clear need for continuity. Continuity is, however, not the only gain from such a program. There is a synergistic aspect here as well that has also been recognized. Real scientific gains come from having concurrent operation of observatories that operate over a wide range of wavelengths with broad spectroscopic and imaging capability.

I think that NASA's OSSA scientists should be commended for their development and support of the Great Observatories concept, as should those in ESA responsible for the development and support of the Horizon 2000 program. Both these programs reflect the more global thinking that is now seen as an integral part of the planning for Space Science missions. The Great Observatories program is in itself also a driver for consideration of a successor to HST. AXAF will be launched well into the life of HST - and SIRTF (the Space Infra-Red Telescope Facility) even further along. To continue the overlap as HST degrades in $10-15+$ years, we need to plan now for its successor.

Beyond continuity we also have (b) the enhanced power and capabilities that the Son of HST will bring. We can, and must, make substantial gains in resolution, sensitivity and throughput. Scientifically, the case for doing so is extremely strong, as is the political necessity. We must open up the "discovery space". In my view, the way to accomplish these joint goals is with an 8-16 m passively-cooled telescope.

Most of us have thought about or can appreciate the gains to be made with $8-16 \mathrm{~m}$ class telescopes on the ground, and have recognized the power of HST and other space telescopes. To have the flux collecting area of an 8-16 m telescope with the gains in resolution, wavelength coverage, and low background that come from having a cool diffraction-limited telescope in space makes for a remarkable and astonishingly powerful observatory. Let me highlight the major gains:

$-\approx 0.01$ arcsecond (FWHM) images in the visible (3-6x smaller than HST);

- a collecting area that is $10-40 \times$ larger;

- an IR background that is $<1 / 1000$ that from the ground.

\section{Scientific Potential}

It is not difficult to think of programs on which such a telescope would have a dramatic effect. Let me highlight a few:

A. One of the most exciting and highest priority scientific objectives for such a telescope must be the detection and measurement of structure in distant galaxies, particularly those at redshifts $z>1$. There are two characteristics of this telescope that would ensure that we would make dramatic advances in this area:

- Resolving power. A resolution of 10 milliarcseconds (mas) corresponds to $\approx 100 \mathrm{pc}$ resolution in galaxies at any redshift, given the currently accepted cosmological parameters. This is remarkable. This is the resolution with which we see Virgo galaxies from the ground. Such resolution is of particular importance for understanding the evolution of galaxies, since much of the structure in galaxies relevant to this question occurs on length scales of 100 pc to $1 \mathrm{kpc}$. Of course, measurement will be difficult at high $z$ because the galaxies will be faint and often of low surface brightness. But there is no doubt that we would make remarkable gains in our knowledge of galaxy structure and evolution at high redshift.

- Much of the light from galaxies at high $z$ comes out in the near-IR, where the sky is darkest from space. This is in the $2-4 \mu \mathrm{m}$ band between zodiacal scattering and emission. 
The background in the near-IR with a passively-cooled space telescope will be less than $1 / 1000$ that from the ground.

$B$. The same high resolution will be of great value for studying galactic nuclei and other compact structures (e.g., VLBI jets and superluminal clouds). By imaging in the near-UV $(0.3-0.4 \mu \mathrm{m})$, an $8-16 \mathrm{~m}$ should be able to resolve $\approx 0.5 \mathrm{pc}$ at Virgo. This resolution will be particularly important for studying nuclei with complex structure. Such structure is difficult to elucidate cleanly with interferometers. In fact, I think that this observatory will complement and enhance the power of the coming interferometric experiments.

C. The resolution will also prove decisive for astrometry, particularly of faint objects. With current detectors we can centroid routinely to $10^{-2} \mathrm{FWHM}$, and even to $10^{-3} \mathrm{FWHM}$ (as has been demonstrated on the ground), given adequate $S / N$, good sampling and a well-characterized detector. With such capability we could measure the distance to stars directly throughout the galaxy to $10 \mathrm{kpc}$. Also, by measuring the proper motions and radial velocities of objects in circular orbits, we should be able to measure the distances to nearby galaxies directly over a 5-10 yr baseline.

D. Finally, but by no means last - since the list is long! - is the detection of earth-like planets around nearby stars. This would probably be the one of the most important discoveries ever made. But it does offer a huge technical challenge. Angel, Cheng and Woolf (1986) have discussed using an apodized $16 \mathrm{~m}$ telescope to find earth-like planets around the nearest stars. Such planets should be found $\approx 0.25^{\prime \prime}$ from a star at a distance of a few parsecs. It seems just possible to detect such an object at $\approx 10 \mu \mathrm{m}$ with a cooled, apodized $16 \mathrm{~m}$ where the first dark diffraction ring corresponds to the planet's orbit. Then the signature of ozone $\mathrm{O}_{3}$ at $9.5 \mu \mathrm{m}$ can be searched for spectroscopically. This is a tough, tough problem, and whether it is really feasible deserves more study, but it is a tremendous idea and a great challenge.

I have only touched the surface of what is a vast number of outstanding scientific problems that could be tackled with such an observatory.

\section{Performance Goals}

With these scientific goals in mind, let me summarise the desirable characteristics of an 8-16 m space telescope.

- Resolution: < 10 mas at $0.3-0.4 \mu \mathrm{m}$, which is $>50 \times$ better than that usually available from the ground, and $>6 \times$ that of HST.

- Collecting area: $10-40 \times$ HST, making it a particularly powerful spectroscopic system.

- Wavelength coverage: $0.1 \mu \mathrm{m}$ to beyond $10 \mu \mathrm{m}$.

- High sensitivity: the "sky" background would be $\approx 33$ mag per resolution element, a remarkable 10 mag or more fainter than that for ground-based telescopes, and some 4 mag less than that for HST.

- Low IR background: $<10^{-3}$ the background from the ground. Passive cooling to $\approx 100^{\circ} \mathrm{K}$ makes the telescope particularly sensitive in the 2-4 $\mu \mathrm{m}$ dark window.

- Multi-purpose Instrumentation: spectroscopic and imaging systems for the UV-Visible-IR.

- Detector mosaics: large, several-arcminute field with diffraction-limited imaging.

- Stable pointing and tracking to $\ll \mathbf{1 0}$ mas.

- High observational efficiency.

The optical performance of this telescope is crucial. Fortunately, new optical fabrication and polishing techniques (below) promise to greatly improve the quality of optical surfaces. The development and demonstration of such techniques must be given particular attention during the early phases of this program.

We want to take advantage of the high optical performance of this telescope by implementing large area detectors in all wavelength regions. While the availability of large-format, solidstate detectors (CCDs) has been a significant concern during the last decade, there should be significant gains in this area in the coming decade.

The last two points bring us to the issue of the location, i.e., the choice of orbit, for the new generation of space observatories. The use of low-earth orbit has clearly added to the complexity 
of the HST system, and to the overall mission planning and operation. And complexity can be equated with cost. It is my view that we should take advantage of emerging technologies and capabilities, and plan to put the next generation of observatories into geosynchronous orbit. Let me just summarize the advantages of geosynchronous orbits for a mission such as this:

- The elimination of short period thermal cycling, thereby lessening the difficulty of achieving the pointing and tracking requirements.

- The attitude control requirements are minimized, since there is low to zero gravity gradient and aerotorque.

- Easier baffling and more effective passive cooling with the small subtended earth-angle.

- Direct data link, allowing high-speed data transfer and simplified control.

- Greatly simplified program optimization and scheduling.

- High observing efficiency.

- Long integrations.

The technologies that will allow us to consider boosting large payloads $(8+m$ ?) to near-earth orbit, allowing some limited on-orbit assembly, if necessary, and transfer to geosynchronous orbit are the likely development of:

1. HLV - Heavy Lift Vehicle ("Space Truck");

2. Space Station;

3. OTV - the Orbital Transfer Vehicle.

The development of these capabilities will influence the type of observatory we launch, and so we should not let the current limited launch capability condition our thinking regarding orbits - or the size of the payloads.

\section{The Telescope}

Given these capabilities, and the performace goals for this HST successor, what is a reasonable system for conceptual development and technology evaluation? I think that we should consider an observatory based on:

- $16 \mathrm{~m}$ primary (four $8 \mathrm{~m}$ segments?);

- Wavelength coverage of $0.1 \mu \mathrm{m}$ to $>10 \mu \mathrm{m}$;

- Diffraction-limited $(\approx 10$ mas in the visible);

- Passively-cooled $\left(\approx 100^{\circ} \mathrm{K}\right)$;

- Wide-field (several arcminutes);

- Geosynchronous orbit.

Scientifically, the case is very strong for a $16 \mathrm{~m}$ telescope. A plausible approach might be to assemble $\approx 8 \mathrm{~m}$-segments on the ground, launch them, and then bring them together to form a $16 \mathrm{~m}$ filled-aperture telescope out of 4 such segments. Such an approach is not beyond likely technology or launch capability developments over the next decade. Segmented mirror technology is being applied to major optical facilities, e.g., the $10 \mathrm{~m}$ Keck telescope (Nelson et al 1984) and the $4 \mathrm{~m}$ LAMP mirror (Aviation Week and Space Technology, Nov 23, 1987). For space we require higher precision in the segment location, but support is easier since there is no gravity and no wind!

Improved optical performance is likely within the next decade. An important development is the dynamic lap approach for polishing optical surfaces. Examples are the membrane approach being developed at Zeiss, and the stressed lap technology being developed by Roger Angel (Angel 1984) and collaborators at Arizona. The techniques should overcome many of the problems associated with CCP (Computer Controlled Polishing) where the small tools leave too much surface structure on scales critical for UV-Visible optics. The new approaches have the potential to give us diffraction-limited performance into the UV.

Passive cooling also seems plausible. The IRAS mirror has settled to $\approx 100^{\circ} \mathrm{K}$, and calculations indicate that a large telescope in geosynchronous orbit could be configured to have its mirrors operate at $\approx 120^{\circ} \mathrm{K}$ (Bely et al 1987). The methods used to polish and test such mirrors do, however, need to be the focus of a significant study and development effort.

It is clear that a project of this magnitude is a costly enterprise. A critical, and integral, part of the technology development will be to evaluate new approaches and technologies whose goal 
will be to minimize cost. This is not an unrealistic goal. Just as the Keck $10 \mathrm{~m}$ and other large ground-based telescopes had to utilize new technology and novel approaches to break the cost scaling laws before they could be considered feasible projects, so we expect that similar advances will lead to a system cost much less than would be suggested by simple scaling from the cost of HST.

\section{Moving Ahead}

I think that a brief review of the history of the HST project will help put the last question "why start now?" into perspective. A workshop was held in 1962 under the auspices of the National Academy of Science. The workshop was titled "A Review of Space Science". It included a recommendation to build a large diffraction-limited space telescope. Yet it was nearly 10 years before the concept was taken up and detailed studies begun on LST, as it was then known. Another five years passed before funding was appropriated and construction started. With launch in 1990 that means that we have seen $25+$ years from a major recommendation to 18 years from detailed design to 12 years for construction and testing.

Now it is realistic to expect that we could move a little faster in the future - but not a whole lot faster. The hurdles are large for any major project. The timescales have been very long, for example, for AXAF. If we are looking to the launch of an HST successor in 15-20 years, now is clearly the time to move.

Where are we today for an 8-16 m observatory? We are a long, long way from our goal. We are at the 1962 stage. Just recently a study was published that was performed under the auspices of the National Academies of Science and of Engineering, and of the Institute of Medicine. The study group was tasked with developing a program for Space Science in the 21st century. The reports of the study groups and an overview volume have recently been published. It is entitled:

Space Science in the Twenty-First Century:

Imperatives for the Decades 1995 to 2015.

The astronomy section contains a recommendation for an 8-16 $\mathrm{m}$ passively-cooled UV-VisibleIR telescope. The first step has been taken, and we need to move on. We have a project that, while ambitious, offers major scientific rewards, is a striking technical challenge, but is not out of the question. Let me quote the report's concluding remark (from the section discussing an 8-16 $m$ telescope in space):

"........ Given a well-directed technology development program, the task group anticipates that an 8- to $16 \mathrm{~m}$ telescope will prove to be within closer reach than a simple extrapolation from HST would suggest."

It is clear that we must move now into a serious evaluation of the scientific, technical, and cost questions and issues, with the goal of defining an 8-16 $\mathrm{m}$ successor to HST within the near future.

\section{Acknowledgements}

I have enjoyed and learnt from stimulating conversations with many people about how to proceed with the next generation of space telescopes. I am particularly grateful to R. Angel, P. Bely, R. Giacconi, and P. Stockman.

\section{References}

Angel, J.R.P. 1984, in Very Large Telescopes, their Instrumentation and Programs, eds. M-H. Ulrich and K. Kjar, ESO, Garching, p.11.

Angel, J.R.P., Cheng, A.Y.S., and Woolf, N.J. 1986, Nature, 322, 341.

Bely, P.Y., Bolton, J.F., Neeck, S., and Tulkoff, P. 1987, Proc. SPIE, 751, 29.

Nelson, J.E., Budiansky, M.P., Gabor, G., and Mast, T.S. 1983, Proc. SPIE, 444, 274. 